\title{
Traditional Uses of Some Wild Plants in Kale and Acıpayam Provinces in Denizli
}

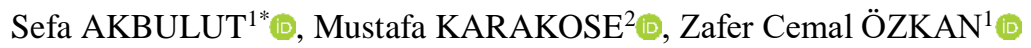 \\ ${ }^{1}$ Karadeniz Technical University, Faculty of Forestry, Department of Forest Engineering, Trabzon, \\ TURKEY \\ ${ }^{2}$ Giresun University, Espiye Vocational School, Giresun, TURKEY \\ *Corresponding Author: sakbulut@ktu.edu.tr
}

Received Date: 02.10.2018

Accepted Date: 01.02.2019

\section{Abstract}

Aim of study: With this study, it was aimed to determine some wild plants in the traditional uses in daily lives by local people for healing, treatment, food, spices, equipment, etc. purposes.

Area of study: The study area was Kale and Acspayam provinces in Denizli between April and October 2016.

Material and Methods: Plant materials were provided from villagers and county of Kale and Acipayam districts. In the identification of plants, "Flora of Turkey and the East Aegean Islands" and "List of Turkish Plants" were used. To determine this, totally 60 people were interviewed face to face. The Informants' Consensus Factor (FIC) and Use Value (UV) formulas were used to analyze the data.

Main results: As a result of the surveys conducted with 60 people, 48 plant taxa belonging to 29 families have been identified. It has been determined that the plants with traditional use in the region are mostly used for the treatment of stomach disorders, respiratory diseases and pain relief. The most used parts of the plants have been leaves. The highest UV is Olea europaea L. var. sylvestris L. (0.65), and the lowest UV is Pulicaria dysenterica (L.) Bernh.. (0.05). The highest FIC value (0.86) is cold and flu.

Research highlights: Although Viscum album subsp. austriacum (Wiesb.) Vollman has been found to be used for different diseases in the literature, its use as a vasodilator drug was first determined by this study.

Keywords: Ethnobotany, traditional uses, herb, Denizli

\section{Kale ve Acıpayam Yöresinde (Denizli) Bazı Doğal Bitkilerin Geleneksel Kullanımları}

$\ddot{O} \mathbf{z}$

Çalışmanın Amacı: Bu çalışma, yerel halkın sağlık, tedavi, gıda, baharat, araç-gereç gibi amaçlarla hangi bitkileri, nasıl kullanıldıklarını saptamak amacıyla yapılmıştır.

Çalışma Alanı: Çalışma 2016 yılı nisan ve ekim ayları arasında Denizli iline bağlı Kale ve Acıpayam ilçelerinde gerçekleştirilmiştir.

Materyal ve Yöntem: Çalışma materyalini Kale ve Acıpayam ilçelerinde yaşayan 60 yerel kişinin gösterdiği, toplanıp teşhis edildiği bitkiler oluşturmaktadır. Bitkilerin teşhisinde "Flora of Turkey and the East Aegean Islands" and "List of Turkish Plants (Vascular Plants) adlı eserlerden yararlanılmıştır. Verilerin analizinde Denek Konsensüs Faktörü (FIC) ve Kullanım Değeri (UV) formülleri kullanılmıştır.

Temel Sonuçlar: 60 kişi ile yapılan anketler sonucunda 29 familyaya ait 48 bitki taksonu teşhis edilmiştir. Yörede geleneksel kullanımı olan bitkilerin daha çok mide rahatsızlıklarının, solunum yolu rahatsızlıklarının tedavisinde ve ağrı kesici olarak kullanıldığı tespit edilmiştir. Bitkilerin en çok kullanılan kısımları ise yaprakları olmuştur. En yüksek UV değeri 0.65 ile Olea europaea L. var. sylvestris L.'e, en düşük UV değeri ise 0.05 ile Pulicaria dysenterica (L.) Bernh.' ya aittir. En yüksek FIC değeri de 0.86 ile nezle ve grip için çıkmıştır.

Araştırma vurguları: Literatürde farklı kullanımları hakkında benzer çalışmalar kayıt edilmiştir. Ancak, bu çalışma ile Viscum album subsp. austriacum (Wiesb.) Vollman'un damar açıcı ilaç olarak kullanımı ilk kez saptanmıştır.

Anahtar Kelimeler: Etnobotanik, geleneksel kullanım, şifalı bitki, Denizli 


\section{Introduction}

Ethnology, composed of ethnos and logos, is a discipline dealing with the distribution, association and activities of different groups of people. The field of activity of the botany is the plants. Botany is a branch of biology and can be defined as the science of plant life. Botany, as well as called plant science, plant biology or phytology. Botany is one of the oldest disciplines in the world. In prehistoric times, people have defined, used and cultivated edible plants for medical purposes. Until the medieval period, herbalists have set up health gardens in many places, especially in the monasteries, in order to raise the plants thought to have healing powers. (Anonymous-1, 2018; Anonymous2 , 2018). Ethnobotanic is one of the most frequently used methods to reveal changes in the cultural values of societies in the modernization process using the plant-human relationship (Heinrich, Bernes, Gibbons \& Williamson, 2004; Kendir and Güvenç, 2010). Ethnobotany is perceived as a term that expresses human-medicinal plant relationships because people often use plants as medicines (Tütenocakl1, 2002).

Utilization of plants and treatment with plants dates back to $3000 \mathrm{BC}$. It is known that many famous Turkish scientists such as Abu Reyhan, Biruni, Ibn-i Sina and Ibn-i Baytar are dealing with herbal drugs (Eşen, 2008).

Because Turkey has a very rich flora and cultural structure, it is an important center in terms of ethnobotany. In recent years there has been a significant increase in such studies (Uysal, Onar, Karabacak \& Çelik, 2010; Polat \& Satıl, 2012; Akaydın, Şimşek, Arrtuluk \& Yeşilada, 2013; Gürdal \& Kültür, 2013; Hayta, Polat \& Selvi, 2014; Mükemre, Behçet \& Cakilcioglu, 2015; Akbulut \& Özkan, 2016).

This study aimed to determine some of wild plants used for local requirements and traditional treatments in the district of Denizli.

\section{Materials and Methods \\ Study area}

The Denizli Province geographically is a gateway between the Aegean, Central Anatolia and Mediterranean Regions, east of the Aegean Region, south-west of the Anatolian peninsula. Denizli city is surrounded by Burdur and Afyon from the east, Muğla from the south, Aydın and Manisa from the west and Uşak from the north. The study area is located between latitudes $37^{\circ} 12^{\prime}-38^{\circ} 12^{\prime} \mathrm{N}$ and longitude $28^{\circ}$ $30^{\prime}-29^{\circ} 30^{\prime}$ E (Figure 1) (Anonymous-3, 2017). It falls within the southern part of $B 2$ and the northern part of $\mathrm{C} 2$ grid square according to grid system of Turkey (Davis, 1965). 58\% of Denizli forests are productive and $42 \%$ are non-productive forests. The forests in the region are generally composed of calabrian pine, black pine, oak, juniper, cedar and other species (Anonymous, 2017). 


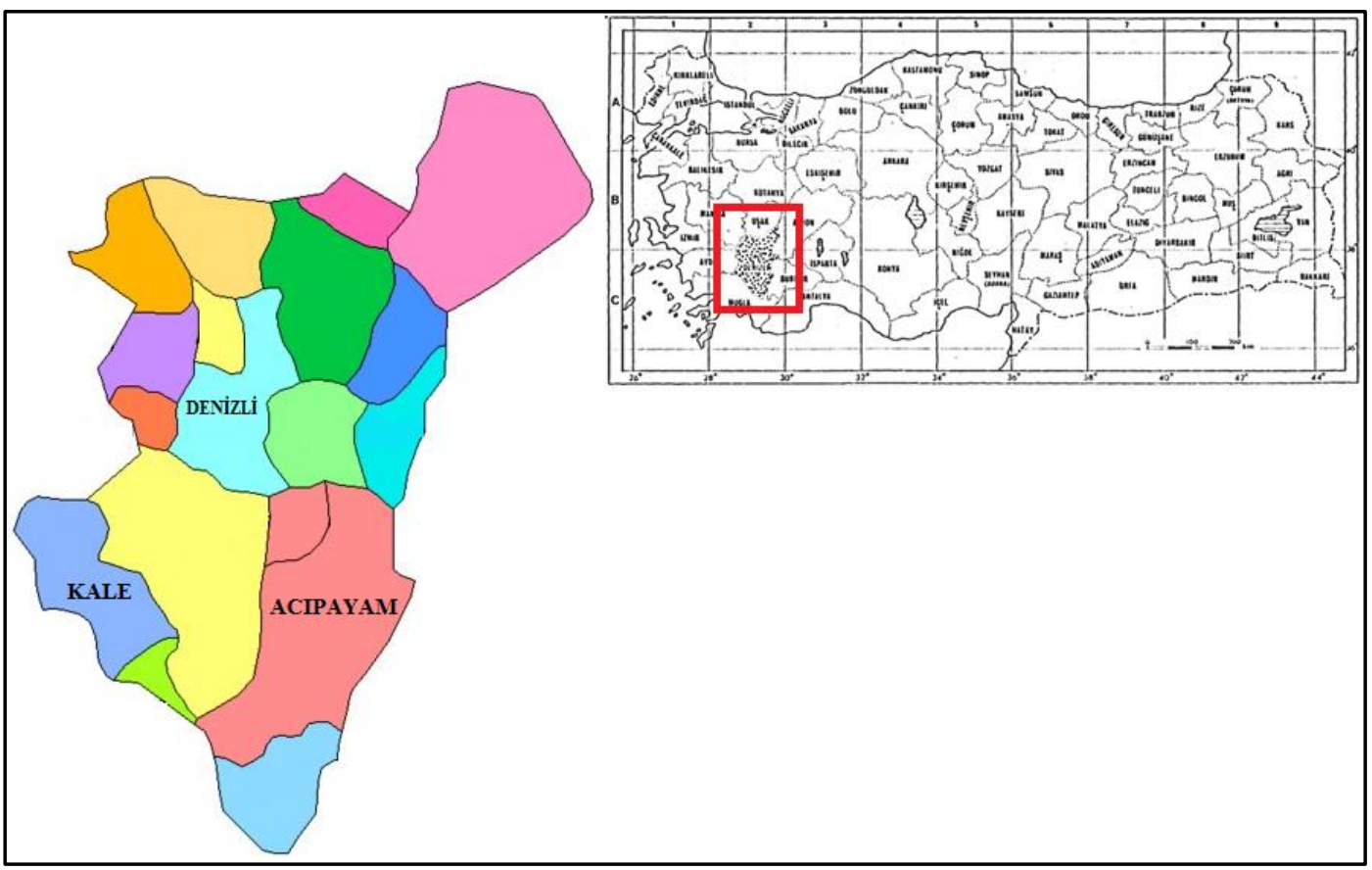

Figure 1. Location of Denizli in grid system of Turkey (Davis, 1965)

\section{Plant Materials and Informants}

This study was conducted to determine some wild plant species which are used for local requirements and traditional treatments of diseases by local people in Kale and Acıpayam provinces of Denizli. Plant materials were obtained from Acipayam and Kale districts and villages. These plants were identified using the flora list of Turkey (Vascular Plants) (Davis 1965-1985; Davis, Mill \& Tan, 1988; Güner, Özhatay, Ekim \& Baser, 2000; Güner, Aslan, Ekim, Vural \& Babaç, 2012). In the study, a two-part questionnaire was applied to total of 60 informants (Appendix A). The questionnaires were applied face to face. The first part of the questionnaire aimed to determine the demographic characteristics such as age, gender, educational level, occupation. In the second part of the questionnaire, vernacular names, part used and preparation technics, and unwritten uses of the plants were noted.

\section{Data Analysis}

The Informants Consensus Factor (FIC), the first of the equations used in the analysis of the data, is used to determine the homogeneity of the information obtained by the surveys. FIC was developed by Trotter and Logan (1986). FIC value ranges from 0 to 1 , where ' 1 ' indicates the highest level of informant consent

$$
\mathrm{FIC}=\text { Nur }-\mathrm{Nt} /(\mathrm{Nur}-1)
$$

Nur, the number of references in each group. Nt is the number of plants utilized (Trotter and Logan, 1986).

Another parameter used for data analysis is "Use Value (UV)". It is used to determine the value of UV (Use Value) that quantitatively expresses the importance of local use of plants:

$\mathrm{UV}=\mathrm{U} / \mathrm{N}$

$\mathrm{U}$ is the number of references per species and $\mathrm{N}$ is the number of people who say the species is used (Trotter and Logan, 1986; Albuquerque, Lucena, Montero, Florentino \& Almeida, 2006; Abe and Ohtani, 2013).

\section{Results and Discussions}

A total 60 informants (27 male, 33 female) were interviewed. The average age of the informants is 57. Some demographic characteristics of the informants are given in Table 1.

A total of 48 taxa belonging to 29 families were identified in the study. Lamiaceae (9), Rosaceae (6), and Poaceae (4) are the most used families. The most preferred methods of preparation are infusion (21), fresh (14), and decoction (10). The most commonly used parts of plants were leaves. The leaves are 
followed by flowers and fruit respectively. The results obtained from the study are given in Table 2.

Table 1. Demographic features of the informants

\begin{tabular}{lcc}
\hline Features & $\begin{array}{c}\text { Number of } \\
\text { informants }\end{array}$ & $\begin{array}{c}\text { Percentage } \\
(\%)\end{array}$ \\
\hline Gender & & \\
\hline Male & 27 & 45 \\
Female & 33 & 55 \\
\hline Educational level & & \\
\hline Illiterate & 3 & 5.0 \\
Primary school & 17 & 28.3 \\
Middle school & 16 & 26.7 \\
High school & 20 & 33.3 \\
University & 4 & 6.7 \\
\hline Age Groups & & \\
\hline $30-49$ & 17 & 28.3 \\
50-69 & 33 & 55.0 \\
$>69$ & 10 & 16.7 \\
\hline Occupation & & \\
\hline Worker & 8 & 13.3 \\
Farmer & 23 & 38.3 \\
Artisan & 6 & 10.0 \\
Officer & 5 & 8.3 \\
Retired & 10 & 16.7 \\
Housewife & 8 & 13.3 \\
\hline
\end{tabular}

According to the results of the study, plants are mostly used to treatment of cold and flu, respiratory tract diseases, stomach disorders, digestive system disorders, laxative, diuretic, painkiller, skin disorders, vascular disorder. The species used mostly for the treatment of stomach disorders were Arum dioscoridis Sm., Liquidambar orientalis Mill., Matricaria chamomilla L. var. recutita (L.) Grierson, Berberis crataegina DC., Hypericum perforatum L., Lavandula angustifolia Mill., Mentha pulegium L., Sideritis libanotica Labill., Thymus longicaulis C.Pres, Rubus canescens DC. (Table 2). Similar results were found in the studies in the ethnobotanical field in Turkey (Everest and Oztürk, 2005; Ezer and Mumcu Arısan, 2006; Koçyiğit and Özhatay, 2006; Kültür, 2007; Uysal et al., 2010; Polat and Sat1l, 2012; Tahri, El Basti, Zidane,
Rochdi \& Douira, 2012; Akbulut and Bayramoğlu, 2013; Gürdal and Kültür, 2013; Akyol and Altan, 2013; Mükemre et al., 2015).

The results of the study showed that some plants used for food in Denizli were used in different regions in a similar way (Arum dioscoridis Sm., Sinapis arvensis L., Malva sylvestris L., Rumex tuberosus L. subsp. L. tuberosus, Crataegus azarolus L. var. azarolus, Rosa canina L., Rubus canescens DC., Rubus idaeus L.) (Uysal et al., 2010; Öztürk, 2006; Dogan, Baslar, Ay \& Mert, 2004; Sağıroğlu, Dalgıç \& Toksoy, 2013; Akaydın et al., 2013; Özçelik and Balabanlı, 2005; Akyol and Altan, 2013; Sargin, Selvi \& Lopez, 2015; Ertuğ, Tümen, Çelik \& Dirmenci, 2004; Akbulut and Özkan, 2016).

Although Arum dioscoridis $\mathrm{Sm}$. is poisonous, it is one of the species used for cooking in the region. The plant is thoroughly cooked in water before it is eaten. In the Eastern Black Sea region, the roots of this plant are consumed in a similar way (Akbulut and Özkan, 2016).

In the present study, Viscum album subsp. austriacum (Wiesb.) Vollman was used to treat vasodilator. According to the literature analysis, the use of Viscum album subsp. austriacum as a vasolidator was recorded for the first time in this study (Bulut, Bozkurt \& Tuzlac1, 2017; Akbulut and Özkan, 2016; Polat and Sat1l, 2012; Kumar, Paul \& Anand, 2009).

It was determined in the study that Arundo donax L. was used for making stick and arbour. Tamarix smyrnensis Bunge was used for making saddle by the local people.

Echinophora tenuifolia subsp. sibthorpiana (Guss.) Tutin is a plant that is often used in making "tarhana", a soup that is unique to the region.

Salvia officinalis L., Alcea rosea L., and Morus nigra $\mathrm{L}$. are exotic taxa that are not a part of the flora of Turkey. However, Salvia officinalis L. and Morus nigra L. are cultured and traded. 
Table 2. Traditional uses of plants in Denizli

\begin{tabular}{|c|c|c|c|c|c|c|}
\hline Family & Botanical name & $\begin{array}{l}\text { Vernacular } \\
\text { name }\end{array}$ & Part used & Preparations & Traditional uses & UV \\
\hline Araceae & $\begin{array}{l}\text { Arum dioscoridis } \\
\text { Sm. }\end{array}$ & Yılan bıçağ & Leaves & $\begin{array}{l}\text { Infusion, } \\
\text { juice }\end{array}$ & $\begin{array}{l}\text { Pass a kidney } \\
\text { stone, } \\
\text { stomachache, } \\
\text { heartburn, food, } \\
\text { eczema (externally } \\
\text { juice) }\end{array}$ & 0.20 \\
\hline Altinginaceae & $\begin{array}{l}\text { Liquidambar } \\
\text { orientalis Mill. }\end{array}$ & $\begin{array}{l}\text { Günlük, } \\
\text { Buhur ağacı }\end{array}$ & Styrax oil & $\begin{array}{l}\text { Internally oil } \\
\text { with honey } \\
\text { and butter }\end{array}$ & Stomach diseases & 0.08 \\
\hline Apiaceae & $\begin{array}{l}\text { Echinophora } \\
\text { tenuifolia subsp. } \\
\text { sibthorpiana } \\
\text { (Guss.) Tutin }\end{array}$ & $\begin{array}{l}\text { Çörtlük, } \\
\text { Tarhana otu }\end{array}$ & $\begin{array}{l}\text { Flowering } \\
\text { and leafy } \\
\text { shoots }\end{array}$ & Dried & $\begin{array}{l}\text { Tarhana preparing, } \\
\text { aromatizer, pickle } \\
\text { making, prevents } \\
\text { fermenting in } \\
\text { pickles }\end{array}$ & 0.32 \\
\hline Apocynaceae & $\begin{array}{l}\text { Nerium oleander } \\
\text { L. }\end{array}$ & Zakkum & $\begin{array}{l}\text { Flowers, } \\
\text { leaves }\end{array}$ & $\begin{array}{l}\text { Decoction } \\
\text { (mixed with } \\
\text { olive oil or } \\
\text { tobacco sap) }\end{array}$ & $\begin{array}{l}\text { Sarcopticide } \\
\text { (externally), } \\
\text { muscicide (for } \\
\text { tobacco field) }\end{array}$ & 0.15 \\
\hline Asteraceae & $\begin{array}{l}\text { Matricaria } \\
\text { chamomilla L. } \\
\text { var. recutita (L.) } \\
\text { Grierson }\end{array}$ & Babatça & $\begin{array}{l}\text { Flowers, } \\
\text { leaves }\end{array}$ & Infusion & Stomach disorders & 0.23 \\
\hline Asteraceae & $\begin{array}{l}\text { Pulicaria } \\
\text { dysenterica (L.) } \\
\text { Bernh. }\end{array}$ & Pireotu & Flowers & Decoction & $\begin{array}{l}\text { Decoction is } \\
\text { mixed with olive } \\
\text { oil and henna. Hair } \\
\text { dye is obtained. }\end{array}$ & 0.05 \\
\hline Berberidaceae & $\begin{array}{l}\text { Berberis } \\
\text { crataegina DC. }\end{array}$ & Kadıntuzluğu & $\begin{array}{l}\text { Fruit, } \\
\text { leaves }\end{array}$ & $\begin{array}{l}\text { Fresh, } \\
\text { infusion }\end{array}$ & $\begin{array}{l}\text { Fresh fruits } \\
\text { regulate blood } \\
\text { circulation, } \\
\text { decrease blood } \\
\text { pressure. Leaf tea } \\
\text { strengthens the } \\
\text { stomach. Leaves } \\
\text { are used in making } \\
\text { salads. }\end{array}$ & 0.23 \\
\hline Brassicaceae & $\begin{array}{l}\text { Armoracia } \\
\text { rusticana (Lam.) } \\
\text { P.Gaertn., } \\
\text { B.Mey. \& } \\
\text { Schreb. }\end{array}$ & Bayır turpu & Root & $\begin{array}{l}\text { Juice (with } \\
\text { honey) }\end{array}$ & Body resistance & 0.07 \\
\hline Brassicaceae & $\begin{array}{l}\text { Sinapis arvensis } \\
\text { L. }\end{array}$ & Hardal & Leaves & $\begin{array}{l}\text { Fresh, } \\
\text { cooking }\end{array}$ & Foodstuff & 0.30 \\
\hline Cupressaceae & $\begin{array}{l}\text { Juniperus } \\
\text { oxycedrus L. }\end{array}$ & Katran ardicı & $\begin{array}{l}\text { Wood, } \\
\text { cone }\end{array}$ & $\begin{array}{l}\text { Extraxt } \\
\text { (mixed } \\
\text { water) }\end{array}$ & Blood purifier & 0.38 \\
\hline Cyperaceae & Carex L. & Kindira & Root & Decoction & Diuretic & 0.12 \\
\hline Euphorbiaceae & Euphorbia L. & Sütleğen & Latex & Externally & Wart & 0.22 \\
\hline Fabaceae & $\begin{array}{l}\text { Glycyrrhiza } \\
\text { glabra L. }\end{array}$ & Biyan & Root & Decoction & $\begin{array}{l}\text { Bronchitis, } \\
\text { antitussive }\end{array}$ & 0.33 \\
\hline Fagaceae & $\begin{array}{l}\text { Quercus } \\
\text { coccifera L. }\end{array}$ & Meşe Peliti & Flowers & Fresh, dried & $\begin{array}{l}\text { Milk yield for } \\
\text { livestock }\end{array}$ & 0.43 \\
\hline Hypericaceae & $\begin{array}{l}\text { Hypericum } \\
\text { perforatum } \mathrm{L} .\end{array}$ & $\begin{array}{l}\text { Sar1 } \\
\text { Kantaron }\end{array}$ & $\begin{array}{l}\text { Flowers, } \\
\text { dried } \\
\text { shoots }\end{array}$ & Infusion, oil & $\begin{array}{l}\text { Stomach disorders, } \\
\text { cancer, painkiller }\end{array}$ & 0.48 \\
\hline
\end{tabular}


Table 2. (continued)

\begin{tabular}{|c|c|c|c|c|c|c|}
\hline Lamiaceae & $\begin{array}{l}\text { Lavandula } \\
\text { angustifolia Mill. }\end{array}$ & Lavanta & Flowers & Infusion & $\begin{array}{l}\text { Tranquillizer, } \\
\text { stomachache }\end{array}$ & 0.18 \\
\hline Lamiaceae & $\begin{array}{l}\text { Mentha } \\
\text { pulegium L. }\end{array}$ & Nane & Leaves & Decoction & $\begin{array}{l}\text { Stomach diseases, } \\
\text { qualm }\end{array}$ & 0.63 \\
\hline Lamiaceae & $\begin{array}{l}\text { Origanum onites } \\
\text { L. }\end{array}$ & Deli kekik & $\begin{array}{l}\text { Flowers, } \\
\text { leaves }\end{array}$ & Infusion & $\begin{array}{l}\text { Cold and flu, } \\
\text { against rise of the } \\
\text { blood sugar level }\end{array}$ & 0.22 \\
\hline Lamiaceae & $\begin{array}{l}\text { Rosmarinus } \\
\text { officinalis L. }\end{array}$ & Biberiye & Leaves & Infusion & $\begin{array}{l}\text { Cold, headache, } \\
\text { migraine }\end{array}$ & 0.15 \\
\hline Lamiaceae & $\begin{array}{l}\text { Salvia officinalis } \\
\text { L. }{ }^{*} \text { e }\end{array}$ & Adaçayı & Leaves & Infusion & $\begin{array}{l}\text { Throat ache, } \\
\text { tonsilitis, } \\
\text { pyorrhoea, } \\
\text { carminative, anti- } \\
\text { nausea, diuretic, } \\
\text { sweat off }\end{array}$ & 0.48 \\
\hline Lamiaceae & $\begin{array}{l}\text { Sideritis arguta } \\
\text { Boiss. \& Heldr. }\end{array}$ & Dağ çayı & $\begin{array}{l}\text { Leaves, } \\
\text { flowers }\end{array}$ & Infusion & $\begin{array}{l}\text { Digestive } \\
\text { disorders, } \\
\text { rheumatism pains, } \\
\text { antitussive, sore } \\
\text { throat, cold and flu }\end{array}$ & 0.32 \\
\hline Lamiaceae & $\begin{array}{l}\text { Sideritis } \\
\text { libanotica Labill. }\end{array}$ & Dağ Çayı & $\begin{array}{l}\text { Leaves, } \\
\text { flowers }\end{array}$ & Infusion & $\begin{array}{l}\text { Cold and flu, } \\
\text { stomachache }\end{array}$ & 0.32 \\
\hline Lamiaceae & $\begin{array}{l}\text { Teucrium } \\
\text { chamaedrys L. }\end{array}$ & Dalakotu & $\begin{array}{l}\text { Flowering } \\
\text { shoots }\end{array}$ & Infusion & $\begin{array}{l}\text { Relieve the } \\
\text { abdominal pain, } \\
\text { haemorrhoids }\end{array}$ & 0.17 \\
\hline Lamiaceae & $\begin{array}{l}\text { Thymus } \\
\text { longicaulis } \\
\text { C.Pres }\end{array}$ & Kekik & $\begin{array}{l}\text { Leaves, } \\
\text { flowers }\end{array}$ & Decoction & $\begin{array}{l}\text { Stomach } \\
\text { discomfort, } \\
\text { prostate, painkiller, } \\
\text { constipation }\end{array}$ & 0.40 \\
\hline Malvaceae & Alcea rosea L. ${ }^{e}$ & Devegülü & $\begin{array}{l}\text { Seeds, } \\
\text { leaves }\end{array}$ & Infusion & $\begin{array}{l}\text { Expectorant, } \\
\text { antitussive, cold, } \\
\text { bronchitis, against } \\
\text { throat, mouth and } \\
\text { teeth } \\
\text { inflammations }\end{array}$ & 0.07 \\
\hline Malvaceae & $\begin{array}{l}\text { Malva sylvestris } \\
\text { L. }\end{array}$ & Ebegümeci & $\begin{array}{l}\text { Leaves, } \\
\text { flowers }\end{array}$ & $\begin{array}{l}\text { Decoction, } \\
\text { fry }\end{array}$ & $\begin{array}{l}\text { Anti- } \\
\text { inflammatory, } \\
\text { food }\end{array}$ & 0.48 \\
\hline Moraceae & Morus nigra L.* & Karadut & $\begin{array}{l}\text { Leaves, } \\
\text { root bark, } \\
\text { fruit }\end{array}$ & Infusion & $\begin{array}{l}\text { Chlorothiazide, } \\
\text { gingiva diseases, } \\
\text { chapped lips }\end{array}$ & 0.46 \\
\hline Myrtaceae & $\begin{array}{l}\text { Myrtus } \\
\text { communis L. }\end{array}$ & Mersin & $\begin{array}{l}\text { Flowers, } \\
\text { Leaves }\end{array}$ & $\begin{array}{l}\text { Fresh, } \\
\text { decoction }\end{array}$ & $\begin{array}{l}\text { Skin disorders, } \\
\text { diarrhea, spices }\end{array}$ & 0.28 \\
\hline Nitrariaceae & $\begin{array}{l}\text { Peganum } \\
\text { harmala L. }\end{array}$ & Nazarotu & Seeds & Amulet & $\begin{array}{l}\text { For religious } \\
\text { beliefs }\end{array}$ & 0.08 \\
\hline Oleaceae & $\begin{array}{l}\text { Olea europaea } \\
\text { L. var. sylvestris } \\
\text { L.* }\end{array}$ & Zeytin & Leaves & Decoction & $\begin{array}{l}\text { Diabetes, } \\
\text { chlorothiazide }\end{array}$ & 0.65 \\
\hline Papaveraceae & $\begin{array}{l}\text { Papaver rhoeas } \\
\text { L. }\end{array}$ & Gelincik & $\begin{array}{l}\text { Flowers, } \\
\text { leaves }\end{array}$ & Cooking & $\begin{array}{l}\text { Galactagogue, } \\
\text { gynaecological } \\
\text { diseases }\end{array}$ & 0.13 \\
\hline Poaceae & Arundo donax L. & Karg1 & Stem & Fresh, dried & $\begin{array}{l}\text { Used for making } \\
\text { stick and arbour }\end{array}$ & 0.40 \\
\hline Poaceae & $\begin{array}{l}\text { Echinochloa } \\
\text { crus-galli (L.) P. } \\
\text { Beauv. }\end{array}$ & Darican & $\begin{array}{l}\text { Parts } \\
\text { plant }\end{array}$ & Fresh, dried & Animal feed & 0.17 \\
\hline
\end{tabular}


Table 2. (continued)

\begin{tabular}{|c|c|c|c|c|c|c|}
\hline Poaceae & $\begin{array}{l}\text { Elymus } \\
\text { flaccidifolius } \\
\text { (Boiss. \& Heldr.) }\end{array}$ & Ayrikotu & Root & Infusion & $\begin{array}{l}\text { Prostate, blood } \\
\text { purifier }\end{array}$ & 0.10 \\
\hline Poaceae & $\begin{array}{l}\text { Melderis } \\
\text { Sorghum } \\
\text { halepense (L.) } \\
\text { Pers. var. } \\
\text { halepense (L.) } \\
\text { Pers. } \\
\text { Rumex }\end{array}$ & Kaynaş & $\begin{array}{l}\text { Parts of } \\
\text { plant }\end{array}$ & Dried & Animal feed & 0.10 \\
\hline Polygonaceae & $\begin{array}{l}\text { Rumex } \\
\text { tuberosus L. } \\
\text { subsp. L. } \\
\text { tuberosus }\end{array}$ & Kuzukulak & $\begin{array}{l}\text { Leaves, } \\
\text { flowers }\end{array}$ & $\begin{array}{l}\text { Decoction, } \\
\text { cooking }\end{array}$ & $\begin{array}{l}\text { Digestive system } \\
\text { disease, food }\end{array}$ & 0.20 \\
\hline Portulacaceae & $\begin{array}{l}\text { Portulaca } \\
\text { oleracea L. }\end{array}$ & Semizotu & Leaves & Fresh & $\begin{array}{l}\text { Lose weight, } \\
\text { laxative }\end{array}$ & 0.37 \\
\hline Rhamnaceae & $\begin{array}{l}\text { Ziziphus jujuba } \\
\text { Mill. }\end{array}$ & Hünnap & Fruits & $\begin{array}{l}\text { Fresh, } \\
\text { infusion }\end{array}$ & $\begin{array}{l}\text { Diabetes, chest } \\
\text { pain, diuretic, } \\
\text { diarrhea and } \\
\text { laxative }\end{array}$ & 0.45 \\
\hline Rosaceae & $\begin{array}{l}\text { Amygdalus webbii } \\
\text { Spach }\end{array}$ & $\begin{array}{l}\text { Badem, } \\
\text { payam }\end{array}$ & $\begin{array}{l}\text { Fruits, } \\
\text { seeds }\end{array}$ & Fresh & $\begin{array}{l}\text { Blood glucose value } \\
\text { and cholesterol } \\
\text { level regulator, } \\
\text { antitussive }\end{array}$ & 0.30 \\
\hline Rosaceae & $\begin{array}{l}\text { Crataegus } \\
\text { azarolus L. var. } \\
\text { azarolus }\end{array}$ & Alıç & $\begin{array}{l}\text { Fruits, } \\
\text { leaves }\end{array}$ & $\begin{array}{l}\text { Infusion, } \\
\text { fresh, syrup }\end{array}$ & $\begin{array}{l}\text { Headache, digestive } \\
\text { system diseases, } \\
\text { food }\end{array}$ & 0.18 \\
\hline Rosaceae & $\begin{array}{l}\text { Pyrus } \\
\text { elaeagnifolia } \text { Pall. }\end{array}$ & Çöğür armudu & Fruits & Fresh & $\begin{array}{l}\text { Blood glucose } \\
\text { regulator, cardio } \\
\text { protective, laxative, } \\
\text { pass a kidney stone, } \\
\text { diuretic, digestive }\end{array}$ & 0.27 \\
\hline Rosaceae & Rosa canina $\mathrm{L}$. & Kuşburnu & Fruits & $\begin{array}{l}\text { Infusion, } \\
\text { marmalade }\end{array}$ & Cold, food & 0.42 \\
\hline Rosaceae & $\begin{array}{l}\text { Rubus canescens } \\
\text { DC. }\end{array}$ & Böğürtlen & Root, fruits & Infusion & $\begin{array}{l}\text { Diuretic, anti- } \\
\text { inflammatory, } \\
\text { stomach discomfort, } \\
\text { food }\end{array}$ & 0.18 \\
\hline Rosaceae & Rubus idaeus L. & Ahududu & $\begin{array}{l}\text { Fruits, } \\
\text { leaves }\end{array}$ & $\begin{array}{l}\text { Fresh, } \\
\text { infusion }\end{array}$ & $\begin{array}{l}\text { Food, gingiva } \\
\text { diseases }\end{array}$ & 0.20 \\
\hline Santalaceae & $\begin{array}{l}\text { Viscum album } \\
\text { subsp. austriacum } \\
\text { (Wiesb.) Vollman }\end{array}$ & Burç, hurç & Leaves & $\begin{array}{l}\text { Infusion, } \\
\text { fresh }\end{array}$ & Vasodilator, fodder & 0.12 \\
\hline Tamaricaceae & $\begin{array}{l}\text { Tamarix } \\
\text { smyrnensis Bunge }\end{array}$ & Ilgin & Branches & Fresh & Saddle & 0.42 \\
\hline Ulmaceae & $\begin{array}{l}\text { Celtis tournefortii } \\
\text { Lam. }\end{array}$ & Çitlık & $\begin{array}{l}\text { Thin } \\
\text { branches }\end{array}$ & Bracelet & Used against nazara & 0.13 \\
\hline Verbenaceae & $\begin{array}{l}\text { Vitex agnus- } \\
\text { castus L. }\end{array}$ & Ayit, Hayit & $\begin{array}{l}\text { Branches, } \\
\text { leaves, } \\
\text { fruit }\end{array}$ & $\begin{array}{l}\text { External, } \\
\text { infusion }\end{array}$ & $\begin{array}{l}\text { Making basket, } \\
\text { antifebrile, fruit } \\
\text { ripening, diuretic, } \\
\text { carminative, } \\
\text { sedative, intestinal } \\
\text { regulator }\end{array}$ & 0.12 \\
\hline
\end{tabular}

*: Cultivated plant in Turkey, e: Egzotic plant 
According to the calculations, Olea europaea L. var. sylvestris L. (0.65), Mentha pulegium L (0.63), Malva sylvestris $\mathrm{L}$. (0.48), Salvia officinalis L. (0.48), Hypericum perforatum L. (0.48), and Morus nigra L. (0.46) were reported to be of the highest use value (UV). Peganum harmala L. (0.08), Liquidambar orientalis Mill. (0.08), Alcea rosea L (0.07), Armoracia rusticana (Lam.) P.Gaertn., B.Mey. \& Schreb. (0.07), and Pulicaria dysenterica (L.) Bernh. (0.05) were found to be of the lowest UV value (Table 3). The high UV value of Olea europaea L. var. sylvestris originates from the fact that it is an important commercial product as well as its medicinal properties in the region.

Information on medicinal use of plants with low UV values such as Peganum harmala, Liquidambar orientalis, Alcea rosea, Armoracia rusticana, Pulicaria dysenterica was obtained from people over middle age. The plants with higher UV values (Olea europaea, Mentha pulegium, Morus nigra) are more popular in the middle age group due to their popularity and widespread use in recent years. In addition, the commercial use of these plants is very high.

Table 3. Comparison of UV values

\begin{tabular}{lccccccc}
\hline & \multicolumn{7}{c}{ Use value (UV) } \\
\cline { 2 - 8 } Species & $\begin{array}{c}\text { Present } \\
\text { study }\end{array}$ & $\begin{array}{c}\text { Mükemre } \\
\text { et al. 2015 }\end{array}$ & $\begin{array}{c}\text { Polat } \\
\text { et al. } \\
2013\end{array}$ & $\begin{array}{c}\text { Polat } \\
\text { et al. }\end{array}$ & $\begin{array}{c}\text { Cak1lc1oğlu } \\
\text { et al. }\end{array}$ & $\begin{array}{c}\text { Polat } \\
\text { \& Satıl } \\
2015\end{array}$ & $\begin{array}{c}\text { Tetik } \\
\text { et al. }\end{array}$ \\
& 0.42 & 0.23 & 0.50 & 0.46 & 0.55 & 0.52 & 0.40 \\
\hline Rosa canina L. & 0.63 & 0.21 & 0.30 & - & 0.30 & 0.34 & - \\
Mentha pulegium L. & 0.48 & - & 0.27 & 0.40 & 0.32 & - & - \\
Hypericum perforatum L. & 0.46 & - & 0.31 & 0.23 & - & - & - \\
Morus nigra L. & & & & & & & \\
\hline
\end{tabular}

Comparisons of the plants with the highest UV values compared to the previous studies are given in Table 3.

According to the information obtained from the participants, recorded discomfort issues were collected under 9 headings (Table 4). The FIC values in the study range from 0.53 to 0.86 . "Cold and flu" had the highest FIC rate (0.86) (37 use-reports for 6 plant species). Origanum onites L., Rosmarinus officinalis L., Sideritis arguta Boiss. \& Heldr., Sideritis libanotica Labill., Alcea rosea L., Rosa canina L. were reported to be among the plant remedies indicated for this use.

The high FIC value for cold and flu suggests that the disease is very common in this region because of the very cold and hard winter months. The low FIC value of vascular disorders can be explained by vegetarian diet and overuse of olive oil (Table 4).
Table 4. Informant consensus factor (FIC) for each ailment

\begin{tabular}{lccc}
\hline Ailment categories & $\begin{array}{c}\text { Number } \\
\text { of use } \\
\text { report } \\
\text { (Nur) }\end{array}$ & $\begin{array}{c}\text { Number } \\
\text { of taxa } \\
\text { (Nt) }\end{array}$ & FIC \\
\hline Cold and flu & 37 & 6 & 0.86 \\
$\begin{array}{l}\text { Respiratory tract } \\
\text { diseases }\end{array}$ & 21 & 6 & 0.75 \\
Stomach disorders & 40 & 11 & 0.74 \\
Digestive system & 25 & 8 & 0.71 \\
disorders & & & \\
Laxative & 9 & 4 & 0.63 \\
Diuretic & 13 & 6 & 0.58 \\
Painkiller & 8 & 4 & 0.57 \\
Skin disorders & 12 & 6 & 0.54 \\
Vascular disorder & 18 & 9 & 0.53 \\
\hline
\end{tabular}

\section{Conclusions}

In this study, 48 herbal and aromatic plants belonging to 29 families were determined in the study site. Salvia officinalis, Alcea rosea, and Morus nigra cultivated plants. Olea europaea, another cultivated plant, is also produced in a very large area. These plants provide significant commercial returns on both domestic and international markets. Ethnobotanical 
information is also being considered by fewer people because of commercial concerns. However, Tarhana (Echinophora tenuifolia subsp. sibthorpiana) is a plant which is mostly sold in local markets for food purposes and is considered as a traditional flavor in tourism.

The continuity of ethnobotanical studies is crucial to reveal the new uses of different plants. The results of these studies contribute to many fields such as medicine, pharmaceutical, paint industry and food industry.

\section{Acknowledgements}

This study was presented at IFES (International Forestry \& Environment Symposium) symposium held in Trabzon on 07-10 November 2017.

\section{Appendix A.}

1- Age and sex of the informant.

2- Marital status of the informant.

3- Educational level of the informant.

4- Occupation of the informant.

5- Place of residence of the informant.

6- What are the medical plants you know?

7- For what purposes do you use medical plants?

8- Which parts of plants are you using?

9- How do you prepare the preparation?

10- What is the vernacular name of the plant used?

11- What are the poisonous plants you know? How do you use them?

12- What are the food plants you know? How do you use them?

13- Do you use auxiliary substances when preparing the preparation?

\section{References}

Abe, R. \& Ohtani, K. (2013). An ethnobotanical study of medicinal plants and traditional therapies on Batan Island, the Philippines. Journal of Ethnopharmacology, 145, 554565.

Akbulut, S. \& Bayramoglu, M.M. (2013). The trade and use of some medical and aromatic herbs in Turkey. Studies on Ethno-Medicine, 7(2), 67-77.

Akbulut, S. \& Özkan, Z.C. (2016). HerbalistCustomer Profile in Medicinal and Aromatic Herbs Trade: A Case Study of
Kahramanmaraş, Turkey. Kastamonu University Journal of Forestry Faculty, 16(1), 246-252.

Akaydın, G., Şimşek, I., Arıtuluk, Z.C. \& Yeşilada, E. (2013). An ethnobotanical survey in selected towns of the Mediterranean subregion (Turkey). Turkish Journal of Biology, 37, 230-247.

Akyol, Y. \& Altan, Y. (2013). Ethnobotanical studies in the Maldan Village (Province Manisa, Turkey). Marmara Pharmaceutical Journal, 17, 21-25.

Albuquerque, U.P., Lucena, R.F.P., Montero, J.M., Florentino, A.T.N. \& Almeida, C.F. (2013). Evaluating two quantitative ethnobotanical techniques. Ethnobotany Research and Applications, 4, 51-60.

Anonymous-1 (2018). Botany. Available< https://en.wikipedia.org/wiki/Botany> [Accessed: 03.12.2018]

Anonymous-2 (2018). Botany. Available< https://www.maximumyield.com/definition/26 5/botany> [Accessed: 03.12.2018]

Anonymous-3 (2017). Denizli province environmental situation report for 2015. Available<http://webdosya.csb.gov.tr/db/ced/e ditordosya/Denizli_icdr2016.pdf $>$ [Accessed: 21.07.2017]

Bulut, G., Bozkurt, M.Z. \& Tuzlacı, E. (2017). The preliminary ethnobotanical study of medicinal plants in Uşak (Turkey). Marmara Pharmaceutical Journal, 21(2), 305-310.

Cakilcioglu, U., Khatunb, S., Turkoglu, I. \& Hayta, Ş. (2011). Ethnopharmacological survey of medicinal plants in Maden (ElazigTurkey). Journal of Ethnopharmacology, 137, 469-486.

Davis, P.H. (1965). Flora of Turkey and the east Aegean Islands, Vol. I. Edinburgh: In University Press.

Davis, P.H. (1965-1985). Flora of Turkey and the east Aegean Islands. Vol. I-IX. Edinburgh: In University Press.

Davis P.H., Mill R.R. \& Tan K. (1988). Flora of Turkey and the east Aegean Islands. Vol. X. Edinburgh: In University Press.

Dogan, Y., Baslar, S., Ay, G. \& Mert, H.H. (2004). The use of wild edible plants in Western and Central Anatolia (Turkey). Economic Botany, 58(4), 684-690.

Ertuğ, F., Tümen, G., Çelik, A. \& Dirmenci, T. (2004). Ethnobotanic Inventory Study of Buldan (Denizli). Tübitak Projesi.

Eşen, B. (2008). Ethnobotanical features of Aydinlar village and its surroundings (Erdemli/Mersin). Master Thesis, Selcuk University, Institute of Science and Technology, Konya. 
Everest, A. \& Ozturk, E. (2005). Focusing on the ethnobotanical uses of plants in Mersin and Adana provinces (Turkey). Journal of Ethnobiology and Ethnomedicine, 1(6), 1-6.

Ezer, N. \& Mumcu-Arisan, Ö. (2006). Folk medicines in Merzifon (Amasya, Turkey). Turkish Journal of Botany, 30, 223-230.

Güner, A., Ozhatay, N., Ekim, T. \& Baser, K.H.C. (2000). Flora of Turkey and the east Aegean islands. Vol. XI, Supplement-II. Edinburgh: In University Press.

Güner, A. Aslan, S., Ekim, T., Vural, M. \& Babaç, M.T. (2012). Türkiye Bitkileri Listesi (Damarlı Bitkiler). Nezahat Gökyiğit Botanik Bahçsi ve Flora Araştırmaları Derneği Yayını, İstanbul.

Gürdal, B. \& Kültür, Ş. (2013). An ethnobotanical study of medicinal plants in Marmaris (Muğla, Turkey). Journal of Ethnopharmacology, 146, 113-126.

Hayta, S., Polat, R. \& Selvi, S. (2014). Traditional uses of medicinal plants in Elazı̆ (Turkey). Journal of Ethnopharmacology, 154, 613-623.

Heinrich, M., Barnes, J., Gibbons, S. \& Williamson, E.M. (2004). Fundamentals of pharmacognosy and phytotherapy. Churchill Livingstone, Edinburgh.

Kendir G. \& Güvenç A. (2010). Ethnobotany and a general view of ethnobotanical studies in Turkey. Hacettepe University Journal of the Faculty of Pharmacy, 30(1), 49-80.

Koçyiğit, M. \& Özhatay, N. (2006). Wild plants used as medicinal purpose in Yalova (Northwest Turkey). Turkish Journal of Pharmaceutical Sciences, 3(2), 91-103.

Kumar, M., Paul, Y. \& Anand, V.K. (2009). An Ethnobotanical Study of Medicinal Plants used by the Locals in Kishtwar, Jammu and Kashmir, India. Ethnobotanical Leaflets, 13, 1240-1256.

Kültür, Ş. (2007). Medicinal plants used in Kirklareli Province (Turkey). Journal of Ethnopharmacology, 111, 341-364.

Mükemre, M., Behçet, L. \& Cakilcioglu, U. (2015). Ethnobotanical study on medicinal plants in villages of Çatak (Van-Turkey). Journal of Ethnopharmacology, 166, 361-374.

Özçelik, H. \& Balabanlı, C. (2005). Medical and aromatic plants of Burdur. I. Burdur Symposium, 1127-1136.

Öztürk, M. (2006). The Flora and Etnobotany of Nizip Region (Aksaray). Master Thesis, Selcuk University, Institute of Science and Technology, Konya.

Polat, R., Cakilcioglu, U., Kaltalioğlu, K., Ulusan, M.D. \& Türkmen Z. (2015). An ethnobotanical study on medicinal plants in
Espiye and its surrounding (Giresun-Turkey). Journal of Ethnopharmacology, 163, 1-11.

Polat, R., Cakilcioglu, U. \& Sat1l, F. (2013). Traditional uses of medicinal plants in Solhan (Bingöl-Turkey). Journal of Ethnopharmacology, 148, 951-963.

Polat, R. \& Sat1l, F. (2012). An ethnobotanical survey of medicinal plants in Edremit Gulf (Balikesir - Turkey). Journal of Ethnopharmacology, 139, 626-641.

Sağıroğlu,, M., Dalgıç, S. \& Toksoy, S. (2013). Medicinal plants used in Dalaman (Muğla), Turkey. Academic Journals, 7(28), 20532066.

Sargin, S.A., Selvi, S. \& Lopez, V. (2015). Ethnomedicinal plants of Sarigöl district (Manisa), Turkey. Journal of Ethnopharmacology, 171, 64-84.

Tahri, N., El Basti, A., Zidane, L., Rochdi, A. \& Douira, A. (2012). Ethnobotanical Study of Medicinal Plants in the Province of Settat (Morocco). Kastamonu University Journal of Forestry Faculty, 12(2), 192-208.

Tetik, F., Civelek, S. \& Cakilcioglu, U. (2013). Traditional uses of some medicinal plants in Malatya (Turkey). Journal of Ethnopharmacology, 146, 331-346.

Trotter, R.T. \& Logan, M.H. (1986). Informant census: A new approach for identifying potentially effective medicinal plants. In Plants in indigenous medicine and diet (Edited by L. N. Etkin), Routledge, Bedford Hill, NY.

Tütenocakl1, T. (2002). Ethnobotics of Ayvacık and its surroundings (B1, Çanakkale). Master Thesis, ÇOMÜ, Çanakkale.

Uysal İ., Onar S., Karabacak E. \& Çelik S. (2010). Ethnobotanical aspects of Kapidağ Peninsula (Turkey). Biological Diversity and Conservation, 3(3), 15-22. 